\title{
Discriminating Hand Gesture Motor Imagery Tasks Using Cortical Current Density Estimation
}

\author{
Bradley Edelman, IEEE Student Member, Bryan Baxter, EMBS Student Member, and Bin He, IEEE \\ Fellow
}

\begin{abstract}
Current EEG based brain computer interface (BCI) systems have achieved successful control in up to 3 dimensions; however the current paradigm may be unnatural for many rehabilitative and recreational applications. Therefore there is a great need to find motor imagination (MI) tasks that are realistic for output device control. In this paper we present our results on classifying hand gesture MI tasks, including right hand flexion, extension, supination and pronation using a novel EEG inverse imaging approach. By using both temporal and spatial specificity in the source domain we were able to separate MI tasks with up to $95 \%$ accuracy for binary classification of any two tasks compared to a maximum of only $\mathbf{7 9 \%}$ in the sensor domain.
\end{abstract}

\section{INTRODUCTION}

Recent investigations of brain-computer interface (BCI) technology have shown great promise for the rehabilitation of patients suffering from various neurological impairments. BCIs using electroencephalographic (EEG) signals are founded on identifying the spatio-temporal dynamics of oscillatory activity originating in the sensorimotor cortex. Motor imagery (MI) tasks, in which movement of a particular body part is simply imagined, can induce changes in these oscillations and have been used to successfully control non-invasive BCI systems in up to 3 dimensions [1, 2]. During motor imagery (MI) tasks, rhythmic activity will either increase or decrease in different brain regions; these phenomena are terms event-related synchronization (ERS) and event-related desynchronization (ERD) respectively.

Using this event-related activity and the somatotopic organization of the motor homunculus, BCIs are able to decode the type of MI task being performed. Nevertheless, signals are significantly distorted as they pass through the skull, dura and meningeal layers from the cortex to the scalp, increasing the difficulty in separating these tasks with reliable results [3]. Thus, this smearing of cortical signals has limited BCI technology to being able to use MI tasks in which the corresponding somatotopic regions are far apart, such as imagination the right and left hands, feet and tongue. These MI tasks have been used to control a BCI with partial success; however, they are very unnatural and not realistic to most applications.

This work was supported in part by NIH EB006433, NSF CBET-1264782, NSF DGE-1069104.

Edelman and B. Baxter are with the Department of Biomedical Engineering, University of Minnesota, Minneapolis, MN 55455, USA

B. He is with the Department of Biomedical Engineering and Institute of Engineering in Medicine, University of Minnesota, Minneapolis, MN 55455 , USA
Consequently, there is a great need to drive the MI tasks used in these non-invasive BCIs towards user-centered applications such that realistic goals can be realized $[4,5]$. The aforementioned MI tasks are irrelevant to many rehabilitative and recreational uses of $\mathrm{BCIs}$ and other tasks must be investigated that better represent the functionality of the application. It is this need that motivates the present study to examine EEG traces of hand gesture MI tasks.

Very few studies have explored hand gesture MI tasks and all have reported their highest binary classification accuracies at between $70 \%$ and $80 \%$ for any two tasks $[6,7$, 8]. Furthermore, all of these studies have performed their analyses using only the EEG sensor recordings which may significantly limit the amount of information that can be interpreted. Source analysis using equivalent dipole modeling and cortical current density (CCD) reconstructions has been shown to provide an alternative method for classifying MI tasks with impressive results and should be investigated for this application as well $[9,10,11,12]$.

In the present study, we investigated the ability to successfully discriminate between four MI tasks of the right hand, flexion, extension, supination and pronation. The minimum-norm estimate was used to convert from sensor space into source space to find unique features of each task that may be used in a non-invasive BCI.

\section{II.METHODS}

\section{A. Experimental Setup and Data Acquisition}

Data from three subjects were analyzed in the present study. These subjects were seated in front of a computer screen and instructed to continuously perform right hand motor imagery of flexion, extension, supination or pronation. Each run was composed of either flexion and extension trials or supination and pronation trials with theses pairings intended to emphasize the back and forth motion of the coupled tasks. Trials were randomized within a run for each experimental session. Trials were structured as follows: a "rest" icon appeared on a computer for 3 seconds, followed by 3 seconds of a target indicating the MI task to perform, and finally a "go" cue for 4 seconds upon which the subject performed the specified MI task. 64-channel EEG signals were acquired using a SynAmpsRT amplifier (Neuroscan Compumedics) at a sampling frequency of $1000 \mathrm{~Hz}$. 


\section{B. Data Processing}

EEG recordings were downsampled to $100 \mathrm{~Hz}$, detrended, and band pass filtered between $5 \mathrm{~Hz}$ and $30 \mathrm{~Hz}$ using a zerophase FIR filter. Noisy trials that contained strong artifacts (i.e. jaw clenching, electrode disconnections) were removed by visual inspection. A surface laplacian was then applied to the remaining trials to enhance focal activity surrounding each electrode. Given that the entire trial did not contain information relevant to the MI task, only the data from one second before the task started to one second after the task ended was kept for further analysis.

Trials were separated into four different data sets based on which of the four MI task was being performed. Each data set was decomposed using independent component analysis (ICA) to reveal temporally independent source activation patterns. Only the independent component (IC) which was located over the left (contralateral to right hand MI task) motor cortex and had strong power in the mu band $(8-12 \mathrm{~Hz})$ was kept while the remaining IC's were discarded. For all subjects, only one component fit these requirements, eliminating ambiguous choices.

\section{A. Minimum-Norm Estimate}

ICA is known to extract temporal sources within an EEG dataset while providing a corresponding spatial map; however, ICA does not explicitly solve the EEG inverse problem. In order to achieve both temporal and spatial precision of the brain dynamics responsible for each task, EEG data at each time point was mapped onto a cortical model using the L2 minimum-norm estimate (MNE). The $\mathrm{MNE}$ is a linear operator that estimates the source activity, $x$, when provided with the forward head model, $A$, and the multi-array EEG data, $b$. This can be done using Eq. 1, where $\lambda$ is a regularization parameter and $I$ is the identity matrix [13].

$$
x=\left(A^{T} A+\lambda I\right)^{-1} A^{T} b
$$

MNE's, especially using the L2 norm, often produce diffuse solutions, but given the large cortical representation of the hand, we can also assume dispersed activation patterns in response to the presented MI tasks [14]. Furthermore, other non-linear inverse algorithms are computationally expensive and more sensitive to noise and accurate head modeling [15]. Without subject-specific cortex models the MNE provided more reasonable results for localizing right hand activity. The boundary element method (BEM) was used to generate a realistic head model of a standard brain for inverse computations. The inverse solution was projected onto a cortical mesh composed of discrete dipoles with fixed orientation normal to the surface. As depicted in Figure 1, the MNE transforms our data from $\mathrm{M} \times \mathrm{N}$ electrode potential values to $\mathrm{P} \times \mathrm{N}$ CCD values where $\mathrm{M}$ is the number of EEG sensors, $\mathrm{N}$ is the number of time points, and $\mathrm{P}$ is the number of dipoles in the forward model.

\section{Feature Extraction and Selection}

Since all MI tasks involve imagining the right hand, source dipoles in a region of interest (ROI) on the left motor cortex were isolated for analysis. This effect is displayed in Figure

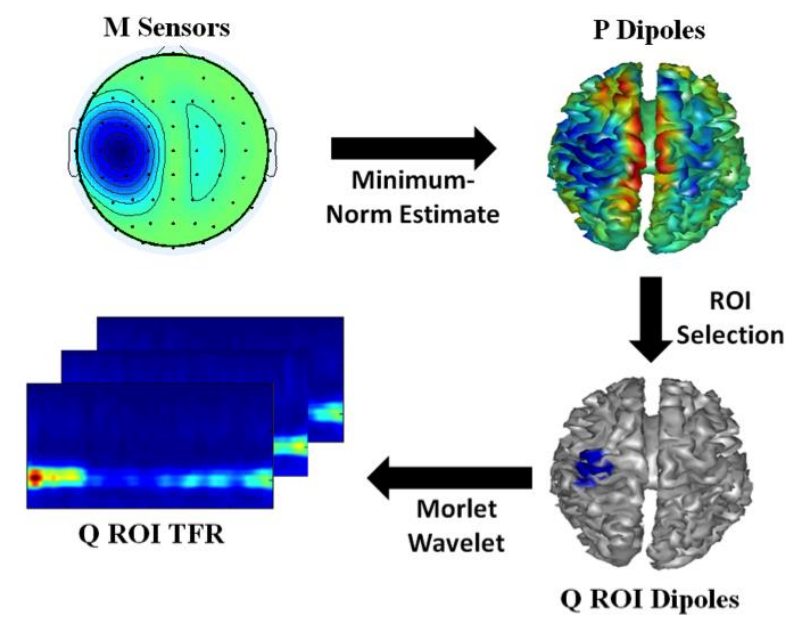

Fig. 1. Event-related activation map for subject one of the extension MI task in sensor space (top left) and its source space transformation (top right). Only those source waveforms within a designated ROI (bottom right) were used for obtaining time-frequency representations (bottom left) to be used for classification (M EEG channels, P total source dipoles, Q ROI source dipoles). This was performed for all MI task data sets.

1 whereby the $\mathrm{P} \times \mathrm{N}$ source data is reduced to $\mathrm{Q} \times \mathrm{N}$ ROI data, where $\mathrm{Q}$ is the number of dipoles in the designated ROI. In this analysis the ROI was defined using general somatotopic knowledge where the hand region of the left motor cortex was estimated. All data was mapped onto the same cortex model, which allowed us to identify dipoles within the ROI prior to analysis. Each dipole corresponds to a row (time series) in the inverse-mapped $\mathrm{P} \times \mathrm{N}$ data matrix. With the ROI pre-defined, the row numbers of interest were already known, making it simple to extract out these time series for further analysis. The C3 electrode waveform was chosen for sensor analysis, as it is known to lie above the cortical region representing right hand control.

All selected dipole and sensor waveforms were extracted and convolved with a Morlet wavelet with a central frequency, $f_{c}$, of $1 \mathrm{~Hz}$ and a Gaussian envelope with a fullwidth half-maximum (FWHM) of $3 \mathrm{sec}$ to obtain a timefrequency representation (TFR) of the activity. Wavelets have the advantage over the Fourier transform by being able to adjust their width in time to capture similar numbers of cycles of varying oscillatory components. To achieve this effect, temporal scales were applied to the mother wavelet (wavelet constructed at $f_{c}$ ) in the form of $\frac{f}{f_{c}}$ where $f$ is the frequency of interest in $1 \mathrm{~Hz}$ intervals between 5 and $30 \mathrm{~Hz}$.

The TFR's were then divided into $2 \mathrm{~Hz}$ frequency bins and $250 \mathrm{~ms}$ time windows for each trial and used as features for classification. The most discriminable features between two data sets were selected based on the mahalanobis distance (MD), a statistical measure of dissimilarity between two groups of data based on their respective feature means and covariances. The single most discriminable feature was first determined by finding the largest MD of any one feature. The second feature selected was that in which, when added to the space spanned by the first, resulted in the largest MD 
of any two features. This process was repeated until the 20 most discriminable features were found between the two data sets and used for classification.

Fisher Linear Discriminant Analysis (LDA) was then applied to the source-estimated and sensor-recorded signals for the four MI tasks. This analysis involved binary classification between all combinations of two different tasks. A 10-fold cross-validation approach was used to test the reliability of the results using $90 \%$ of the trials for training and the remaining $10 \%$ for testing.

\section{RESULTS}

For all subjects, an IC representing each of the four MI tasks was found over the left motor cortex exhibiting strong mu band activity. This result lends confidence that the subjects are in fact performing a MI task involving the right hand. This is important to ensure that we are not attempting to discriminate between a right hand MI task and that of a different body part or between two completely arbitrary tasks. For all binary combinations of the four MI tasks, the proposed source space method resulted in increased classification accuracies over sensor space. The largest average increase was seen in flexion vs. extension at $+20.11 \%$ and the lowest average increase was seen in extension vs. pronation at $+7.62 \%$. Among all binary classifications in all three subjects (6 cases per subject, 18 cases total), only twice did the source method result in a lower accuracy than the sensor method. These two cases were extension vs pronation for both subject 1 and subject 2; however, these decreased accuracies were only $-1.00 \%$ and $2.50 \%$ respectively.

Table 1. Average Fisher LDA results from three subjects comparing sensor (C3) and source (ROI) data for the different MI tasks (E - Extension, F - Flexion, $\mathrm{S}$ - Supination, $\mathrm{P}$ - Pronation).

\begin{tabular}{|c|c|c|}
\hline & Sensor (\%) & Source (\%) \\
\hline $\mathbf{F} / \mathbf{E}$ & $75.70 \pm 3.42$ & $95.82 \pm 4.01$ \\
\hline $\mathbf{F} / \mathbf{S}$ & $74.92 \pm 0.58$ & $87.73 \pm 12.15$ \\
\hline $\mathbf{F} / \mathbf{P}$ & $77.65 \pm 8.96$ & $91.85 \pm 5.97$ \\
\hline $\mathbf{E} / \mathbf{S}$ & $78.96 \pm 4.58$ & $95.73 \pm 5.90$ \\
\hline $\mathbf{E} / \mathbf{P}$ & $78.80 \pm 6.61$ & $86.42 \pm 12.47$ \\
\hline $\mathbf{S} / \mathbf{P}$ & $72.00 \pm 0.74$ & $88.48 \pm 10.16$ \\
\hline
\end{tabular}

\section{DISCUSSION}

Much of the previous BCI research has examined the origin and utility of MI tasks of different limbs to activate distinct spatial regions of the cortex; however, little work has been done to investigate the usability of different functional MI tasks from the same body part. In sensor space, we found that each functional MI tasks activated a similar diffuse region over the sensorimotor cortex. The thousands of dipoles that make up the cortical mesh provide a greatly increased spatial sampling of the brain compared to our 64 channel EEG montage. By incorporating not only this increased sampling but also, anatomical constraints, inverse mapping allows subtle differences between MI tasks in sensor space to become much more apparent in source

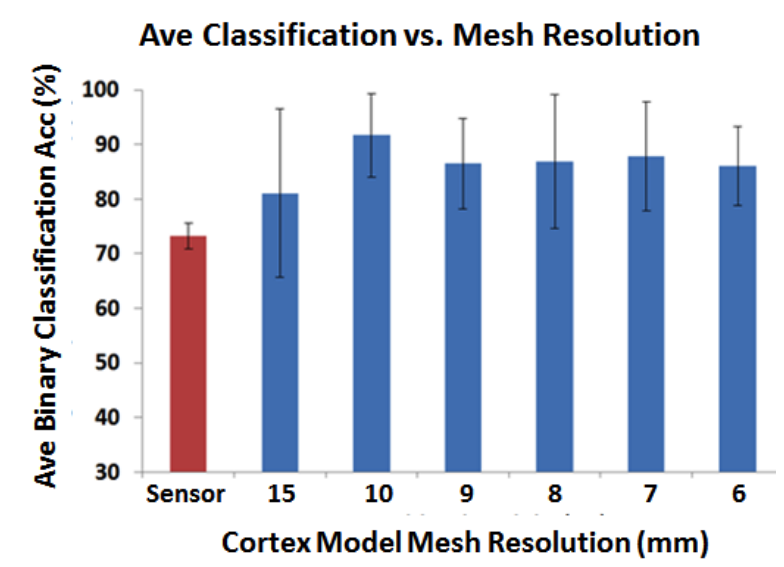

Fig 2. Average source-based binary classification accuracy plotted against cortical mesh resolution. The coarsest mesh is $15 \mathrm{~mm}$ and the finest mesh is $6 \mathrm{~mm}$. In all cases, the source based analysis using the cortical mesh produced better results than sensor analysis.

space. It is with this principle that our source based method provides increased classification accuracies.

To determine the influence of the mesh geometry, we repeated our analysis using varying mesh resolutions for one of the subjects. The average classification accuracies of the six binary combinations of MI tasks are displayed for the different mesh resolutions in Figure 2. In these models the resolution indicates the distance between neighboring nodes in the BEM model and thus $15 \mathrm{~mm}$ indicates the coarsest mesh and $6 \mathrm{~mm}$, the finest. The average accuracy fluctuates between $81.1 \%$ and $91.7 \%$ indicating that the mesh does in fact play a role in the effectiveness of our proposed method; however, even the coarsest mesh produces better results than sensor analysis. We conclude that as long as the classifier is trained and tested with a consistent model, the method does not show significant degenerative effects.

\section{CONCLUSION}

In the present study, we have evaluated the performance of using CCD reconstructions to discriminate neural traces of different right hand gesture MI tasks. The proposed source imaging based technique employs both temporal and spatial task specificity of cortical behavior in order to characterize the brain processes involved with right hand flexion, extension, supination and pronation MI. Our source based method resulted in increased binary classification accuracies between all combinations of tasks for three subjects over sensor based data.

\section{ACKNOWLEDGMENT}

The authors are thankful to Clara Zhang and Long Yu for helpful discussions.

\section{REFERENCES}

[1] D.J. McFarland, W. Sarnacki, and J. Wolpaw, "Electroencephalographic (EEG) control of three-dimensional movement," J of Neural Eng 7:036007, 2010.

[2] K. Lafleur, K. Cassady, A. Doud, K. Shades, E. Rogin, and B. $\mathrm{He}$, "Quadcopter control in three-dimensional space using a 
noninvasive motor-imagery-based brain-computer interface," J of Neural Eng 10:046003, 2013.

[3] B. He, S. Gao, H. Yuan and J. Wolpaw, "Brain Computer Interface," in B. $\mathrm{He}(\mathrm{Ed})$ : Neural Engineering, Springer, pp.87$151,2013$.

[4] D. Plass-Oude Bos, M. Poel, and A. Nijholt, "A study in usercentered design and evaluation of mental tasks for BCI," Proceedings of the $17^{\text {th }}$ International Multimedia Modeling Conference 122-134, 2011

[5] O. Tonet, M. Marinelli, L. Citi, P.M. Rossini, L. Rossini, G. Megali, and P. Dario, "Defining brain-machine interface applications by matching interface performance with device requirements," J of Neurosci Methods 167:91-104, 2008

[6] A. Vuckovik and F. Sepulveda, "Delta band contribution in cue based single trial classification of real and imaginary wrist movements," Med \& Biolog Eng \& Comp 46:529-539, 2008.

[7] A. Vuckovik and F. Sepulveda, "A two-stage four-class bci based on imaginary movements of the left and the right wrist," Medical Eng \& Physics 34:964-971, 2012.

[8] A.K. Mohamed, T. Marwala, and L.R. John, "Single-trial eeg discrimination between wrist and finger movement imagery and execution in a sensorimotor bci," $33^{\text {rd }}$ Annual International Conference of the IEEE EMBS 6289-6293, 2011

[9] B. Kamousi, A. Liu, and B. He, "Classification of motor imagery tasks for brain-computer interface applications by means of two equivalent dipoles analysis," IEEE Transactions on Neural Sys and Rehab Eng 13(2):166-171, 2005.

[10] B. Kamousi, A.N. Amini., and B. He, "Classification of motor imagery by means of cortical current density estimation and von Neumann entropy," J Neural Eng 4:17-25, 2007.

[11] H. Yuan, A. Doud, A. Gururajan, and B. He, "Cortical imaging of event-related (de)synchronization during online control of brain-computer interface using minimum-norm estimates in frequency domain," IEEE Transactions on Neural Sys and Rehab Eng 16(5):425-431, 2008.

[12] L. Qin, L. Ding, and B. He, "Motor imagery classification by means of source analysis for brain computer interface applications," J Neural Eng 2(4):65-72, 2004.

[13] B. He, X. Zhang, J. Lian, H. Sasaki, D. Wu, and V.L. Towle, "Boundary element method-based cortical potential imaging of somatosensory evoked potentials using subjects' magnetic resonance images," NeuroImage 16:564-576, 2002

[14] Grech, R., Cassar, T., Muscat, J., Camilleri, K.P., Fabri, S.G., Zervakis, M., Xanthopoulos, P., Sakkalis, V., Vanrumste, B., “ Review on solving the inverse problem in EEG source analysis," J NeuroEng and Rehab 5:25, 2008

[15] Ding, L., He, B., "Sparse source imaging in electroencephalography with accurate field modeling," Human Brain Mapping 29:1053-1067, 2008 